\title{
TIME DEPENDENCE OF THE RADIATION-INDUCED EPR SIGNAL IN SUCROSE
}

\author{
Marc Desrosiers* and Samara Wadley \\ Ionizing Radiation Division, Physics Laboratory, National Institute of Standards and Technology, \\ Gaithersburg, MD 20899, USA
}

\section{Received August 25 2005, amended October 31 2005, accepted November 232005}

\begin{abstract}
Sucrose and common household sugars (e.g. cane) have been studied as dosemeters for a wide variety of applications. However, previous studies of the post-irradiation time dependence of irradiated sugar did not include an electron paramagnetic resonance (EPR) reference material. This work employs synthetic ruby as an EPR reference material to remove significant spectrometer/environmental influences on the measured time-dependent changes in the EPR spectral amplitude of irradiated sucrose. As such, these more accurate measurements should replace the previously published data.
\end{abstract}

\section{INTRODUCTION}

Sugar is generally considered to possess many attractive dosimetry attributes. Common 'cane' sugar, composed primarily of sucrose, is ubiquitous and has a long-lived radiation-induced free radical that can be quantified from biologically relevant doses up to industrial processing doses. A number of publications have reported on the dosimetric properties of sugars and their use in radiation accident dose reconstruction $^{(1-4)}$. Inconsistencies between these reports were the basis of a cooperative study of table sugar and sucrose by the National Institute of Standards and Technology (NIST) and the Istituto Superiore di Sanità (ISS) $)^{(5)}$. The NIST/ISS study estimated that the minimum measurable dose for cane sugar was between 5 and $10 \mathrm{cGy}$. Other observations included dose-dependent changes in the electron paramagnetic resonance (EPR) spectral features that continued to vary for many hours after irradiation. The time dependencies of these changes were also dependent on the absorbed dose of the sugar. The time dependence of the EPR signal amplitude for 100 Gy irradiated sugar was sigmoidal in the first $24 \mathrm{~h}$ and saturated at $\sim 80 \mathrm{~h}$ post-irradiation up to the end of the recording period (nearly $200 \mathrm{~h}$ ). The sigmoidal behaviour was not observed at lower doses. The EPR signal saturation region for $100 \mathrm{~Gy}$ irradiated sugar was the same for each of the other doses: 10, 1 and 0.2 Gy. For the 10 and 1 Gy irradiated sugar, a distinct minimum in the EPR signal amplitude was observed between 5 and $10 \mathrm{~h}$ postirradiation. This minimum in the post-irradiation EPR signal amplitude time course was nearly identical for sucrose and cane sugar.

Several years after the NIST/ISS sugar dosimetry study, modifications were made to the NIST EPR

*Corresponding author: marcd@nist.gov spectrometer that enabled it to compensate for random short-term fluctuations and long-term drifts in the EPR spectrometer sensitivity ${ }^{(6)}$. The EPR spectrometer is currently equipped with a ruby crystal reference device that, when the sample signal is normalised to its signal amplitude, effectively removes influences on the EPR signal amplitude variation derived from the spectrometer sensitivity and sample moisture content. The time-dependent measurements of irradiated sucrose in previous studies were not recorded with the ruby reference device. This work reports more accurate time dependencies of the EPR signal amplitude of irradiated sucrose with the intent that these data replace the previous measurements ${ }^{(5)}$ that included signal variations from instrumental and environmental influences.

\section{MATERIALS AND METHODS}

The mention of commercial products throughout this paper does not imply recommendation or endorsement by the National Institute of Standards and Technology, nor does it imply that products identified are necessarily the best available for this purpose. Sucrose (99+\%) was purchased from Sigma Chemical (St Louis, MO). The sucrose was sieved; the particle size range selected for this study was $500-850 \mu \mathrm{m}$. The two ${ }^{60} \mathrm{Co}$ irradiators used were calibrated with alanine; the dose rate (in $\mathrm{H}_{2} \mathrm{O}$ ) for the 10 and $100 \mathrm{~Gy}$ irradiations was $24 \mathrm{~Gy} \mathrm{~min}^{-1}$, and for the $1 \mathrm{~Gy}$ irradiation was $0.89 \mathrm{~Gy} \mathrm{~min}^{-1}$ (combined expanded uncertainty $=2 \%, k=2$ ). The irradiated sucrose was transferred to a quartz EPR tube (Wilmad, Buena, NJ) and loaded to a height of $\sim 3 \mathrm{~cm}$ (sufficient to span the height of the EPR microwave resonator) with gentle tapping of the tube on a hard surface to maintain a density as uniform as possible. The sucrose mass in the EPR tube was not measured. For the EPR measurements, 

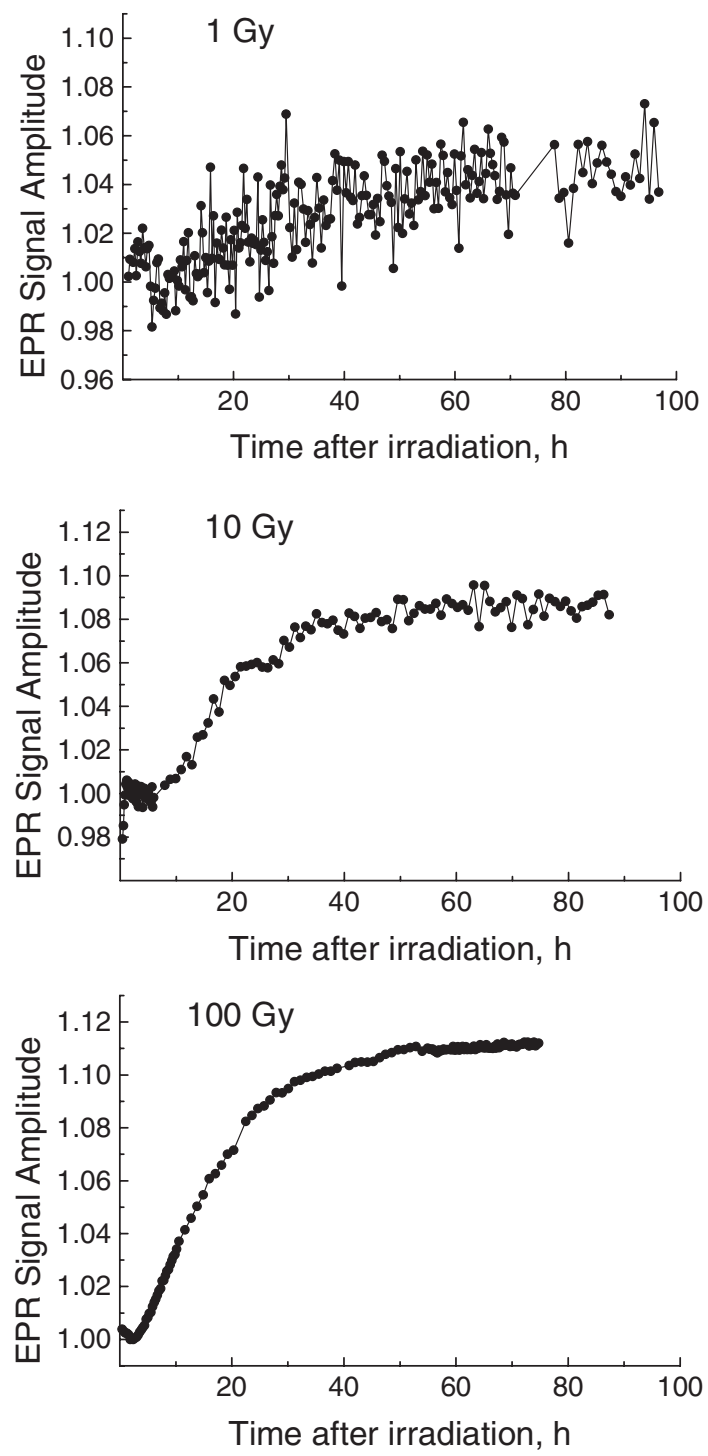

Figure 1. Plots of EPR signal amplitude (normalised to the initial value) vs. time after irradiation for sucrose gammairradiated to $1 \mathrm{~Gy}$ (top), $10 \mathrm{~Gy}$ (middle) and $100 \mathrm{~Gy}$ (bottom).

a Bruker ECS106 EPR spectrometer was used with the EPR parameter settings: microwave frequency, 9.6 GHz; modulation amplitude, $1.0 \mathrm{mT}$; microwave power, $10 \mathrm{~mW}$; magnetic sweep width, $10.0 \mathrm{mT}$; number of spectral scans accumulated, 1. The EPR spectrum was identical to that previously reported ${ }^{(5)}$. The sum of the spectral minimum (absolute value) and maximum was used as the EPR signal amplitude. The sucrose EPR amplitude measured at each time interval was normalised to the amplitude of the ruby EPR signal amplitude measured in tandem with sucrose. The recording conditions and procedure is described in detail in a publication dedicated to the use of the ruby intensity standard ${ }^{(6)}$.

\section{RESULTS AND DISCUSSION}

Figure 1 shows the time dependence for the EPR signal amplitude of 1, 10 and 100 Gy irradiated sucrose. Owing to the relatively weak signal at $1 \mathrm{~Gy}$, the EPR signal amplitude time dependence has considerable signal scatter. An overall increase in the signal amplitude of $\sim 4 \%$ was observed over the nearly $100 \mathrm{~h}$ measurement period. At a dose of 10 Gy an initial decrease $(<1 \%)$ was observed at post-irradiation times $<10 \mathrm{~h}$, followed by an increase in signal amplitude to $\sim 8 \%$ that saturated at $\sim 60 \mathrm{~h}$. For 100 Gy irradiated sucrose, after an initial decrease, an increase in signal amplitude of $\sim 11 \%$ was measured. As observed for $10 \mathrm{~Gy}$, the 100 Gy signal amplitude reached a maximum at $\sim 60 \mathrm{~h}$.

\section{CONCLUSIONS}

EPR measurements of the time dependence of irradiated sucrose were recorded in conjunction with a ruby EPR intensity standard. The ruby-adjusted, time-dependent trends in the signal amplitude are less complex and more consistent from 1 to $100 \mathrm{~Gy}$ than the time dependencies previously reported ${ }^{(5)}$. The inconsistencies with absorbed dose and the sigmoidal nature of the time-dependent signal trends previously measured were probably due to EPR spectrometer sensitivity variations ${ }^{(6)}$. As previously reported, the relative magnitude of the signal amplitude increase is larger at higher doses. The changes observed in the irradiated sucrose time dependence in the 1-10 Gy range are modest and could be taken into account in the use of sucrose as a dosemeter.

\section{ACKNOWLEDGEMENTS}

Ms. Wadley performed this work as a guest researcher from the Southern University at Baton Rouge, Louisiana. This material is based in part upon work supported by the National Science Foundation under Grant No. 9605093. Any opinions, findings and conclusions or recommendations expressed in this material do not necessarily reflect the views of the National Science Foundation.

\section{REFERENCES}

1. Nakajima, T., Otsuki, T., Hara, H., Nishiwaki, Y. and Matsuoka, M. ESR sensitivity comparison of sugar from 
various sources and cavity mode effect. Radiat. Prot. Dosim. 34, 303 (1990).

2. Gutierrez, A. and Azorin, J. Lyoluminescence and electron spin resonance of commercial sugar in accident dosimetry. Nucl. Sci. J. 30, 137 (1993).

3. Tchen, A., Greenstock, C. L. and Trivedi A. The use of sugar pellets in ESR dosimetry. Radiat. Prot. Dosim. 46, 119 (1993).

4. Nakajima, T. Estimation of absorbed dose to evacuees at Pripyat-City using ESR measurements of sugar and exposure rate calculations. Appl. Radiat. Isot. 45, 113 (1994).

5. Fattibene, P., Duckworth, T. and Desrosiers, M. F. Critical evaluation of the sugar-EPR dosimetry system. Appl. Radiat. Isot. 47, 1375-1379 (1996).

6. Nagy, V. Y., Sleptchonok, O. F., Desrosiers, M. F., Weber, R. T. and Heiss, A. H. Advancements in accuracy of the alanine dosimetry system. Part 3: usefulness of an adjacent reference sample. Radiat. Phys. Chem. 59, 429-441 (2000). 